\title{
Dependence of electron beam diameter, electron energy, resist thickness and resist type for forming nano-sized dot arrays in EB lithography by using Monte Carlo Simulation
}

\author{
Hui Zhang ${ }^{1,3, \text { *, Yulong Zhang }}{ }^{2,3}$, Sumio Hosaka ${ }^{2,3}$, You Yin ${ }^{2,3}$ \\ ${ }^{1}$ Advanced Technology Research Center, Gunma University, Japan \\ ${ }^{2}$ Graduate School of Engineering, Gunma, Japan \\ ${ }^{3}$ Gunma University, 1-5-1 Tenjin, Kiryu, Gunma 376-8515, Japan
}

\section{Email address:}

t10802275@gunma-u.ac.jp (H. Zhang), t10811372@gunma-u.ac.jp (Y. Zhang), hosaka@el.gunma-u.ac.jp (S. Hosaka), yinyou@gunma-u.ac.jp (Y. You)

\section{To cite this article:}

Hui Zhang, Yulong Zhang, Sumio Hosaka, You Yin. Monte Carlo Simulation for Nano-sized Dot Arrays in EB Lithography by Using Monte Carlo Simulation. American Journal of Nanoscience and Nanotechnology. Vol. 1, No. 1, 2013, pp. 11-16.

doi: $10.11648 /$ j.nano.20130101.13

\begin{abstract}
We have calculated the electron energy deposition distribution in Calixarene negative resist and analyzed the development profile in order to improve the resolution of pattern. From the trajectories and energy deposition distribution in resist at various beam diameters, it is obvious that the thinner resist film should be adopted for formation of very fine dots. The analysis of relationship between the thickness of resist and dot diameter based on the critical energy densities shows that the thickness of resist less than $20 \mathrm{~nm}$ can obtain 5 -nm size dot pattern at the range of critial energy of 6.25 $\mathrm{keV} / \mathrm{cm}^{3}-56.25 \mathrm{keV} / \mathrm{cm}^{3}$. The simulation of resist development profile indicates that dot size of $3 \mathrm{~nm}$ can even be obtained at a higher critical energy density at $156.25 \mathrm{keV} / \mathrm{cm}^{3}$. Furthermore, Calixarene resist is more suitable than PMMA positive resist by comparison of these two resists.
\end{abstract}

Keywords: Electron Beam Lithography, Gaussian Beam, Monte Carlo Simulation, Energy Deposition Distribution, Resist Profile

\section{Introduction}

The recording density of magnetic data storage is increasing and is expected to exceed $1 \mathrm{Tbit} / \mathrm{in}^{2}$ in the future The formation of nanometer-scale dot arrays is developed for achieving high-density data storage. ${ }^{1-3)}$ It is crucial to develop methods for fabricating high density magnetic nano dot arrays with a pitch of less than $25 \mathrm{~nm}$ and to achieve the next generation of patterned media with a recording density of up to $1 \mathrm{Tbit} / \mathrm{in}^{2}{ }^{2}$ in the future. ${ }^{4-6)}$

Electron beam lithography (EBL) ${ }^{7-10)}$ has been expected as a candidate to form very fine pitch and dot arrays for patterned media. Using the EBL, many efforts have been made to improve the density of data storage. For example, 10 -nm wide line patterning by using p-methy1-methy-lacetoxy-calix[6]arene was first reported by Fujita et al. ${ }^{11)}$ Calixarene resist dot arrays with $20-\mathrm{nm}$ pitch were demonstrated by Hosaka et al. by using a 30
$\mathrm{keV}$ accelerating voltage. ${ }^{12)}$ However, the formation of high-density nano-scale dots is still one of challenging works because the exposure dosage cannot be controlled due to the charging-up ${ }^{13)}$ and proximity effects ${ }^{14}$ ). So far, the calculation of exposure and development processes by using EB drawing with Calixarene resist has not been done yet to analyze the resolution-limiting factors of EBL in the sub-10-nm range, which has limited our ability to further improve its resolution. Therefore, we try to calculate the electron energy deposition distribution in Calixarene resist and analyze the development profile in order to improve the resolution of pattern.

In this paper, we briefly describe our Monte Carlo simulation for the scattering model using a Gaussian beam as an incident electron beam with a diameter of about $2 \mathrm{~nm}$ to investigate the energy deposition distribution (EDD) in thin Calixarene resist. Using the spatial distribution of EDD, we determine the profile of EDD during the electron beam 
scattering process. It is estimated to develop dot sizes at various critical energy densities and to ensure to form fine pattern with high resolution.

\section{Monte Carlo Simulation Model}

\subsection{The Modeling of Gaussian Beam}

In EBL, the EB writing system uses a finely focused Gaussian electron beam for formation of fine pattern. We have to consider the beam profile as an incident electron beam in the simulation. Intensity of Gaussian electron beam can be represented in one dimension by the function ${ }^{15)}$ :

$$
\mathrm{I}(r)=\frac{1}{\sqrt{2 \pi} s} e^{-\frac{r^{2}}{2 s^{2}}}
$$

where $r$ is the distance from a center of the beam, and $s$ is the standard deviation.

For the miniaturization of the dot size, small $s$ value is required. The $s$ value has to be $1 \mathrm{~nm}$ because the advanced SEM has very fine beam with a resolution of about $2 \mathrm{~nm} .{ }^{4}$ We can determine the incident position of primary electron using Monte Carlo simulation. Using the probability $P$ and Eq.(1), the position $r$ is determined by following equations,

$$
\begin{aligned}
\mathrm{V}(r) & =\int_{0}^{r} I(r) \cdot 2 \pi r d r \\
P & =\frac{V(r)}{\int_{0}^{\infty} V(r) d r}
\end{aligned}
$$

where $P$ is a uniform random number between 0 and 1 . We prepared to make a data table of Eq.(2) by every increment of $r$ of $0.2 \mathrm{~nm}$ in a range of the $r$ from 0 to $5 \mathrm{~s}$. The normalized $V(r)$ corresponds to the probability $P$. The $P$ can determine the $r$ by referring the table. And we can use the linear interpolation methods to calculate the $r$. Then we can use the $r$ to calculate the coordinate of first incident point of electron scattering.

\subsection{The Modeling of Electron Scattering Process}

The exposure of the electron to resist in EBL makes energy deposition in the scattering of accelerated electrons in the target. It is based on both a single scattering model and a continuous slowing down approximation. The screened Rutherford formula is used for the differential elastic scattering cross-section as follows, ${ }^{16-19)}$

$$
\frac{d \sigma}{d \Omega}=\frac{e^{4} Z_{i}\left(Z_{i}+1\right)}{4 E^{2}(1-\cos \theta+2 \beta)}
$$

where $\beta$ is the screening parameter, $e$ is the electronic charge, $Z$ is the atomic number of the material, $E$ is the energy of electrons, and the scattering angle $\theta$ can be calculated using the following equations:

$$
\theta=\cos ^{-1}\left(1-\frac{2 \beta R_{1}}{1-\beta-R_{1}}\right)
$$

where the $R_{1}$ is independent equidistributed random number between 0 and 1 .

\subsection{Energy Loss and Energy Deposition Distribution}

Since the electron suffers scattering along its trajectory, it continuously losses its kinetic energy along the trajectory. The Bethe's equation is good empirical in calculating the energy loss in electron atom interaction. The Bethe's approximation is given by ${ }^{20)}$

$$
-\frac{d E}{d s}=\frac{2 \pi e^{4}}{E} \sum n_{i} Z_{i} \ln \left(\frac{1.166 E}{J_{i}}\right)
$$

where the $n_{i}$ is volume density of atoms, the $J_{i}$ is mean ionization energy of atom $i$.

The terminal energy of the $\mathrm{n}^{\text {th }}$ scattering is:

$$
E_{n+1}=E_{n}-\left|\frac{d E}{d s}\right|_{E_{n}} \cdot \Lambda_{n}
$$

where the $E_{n}$ is the energy of the (n-1)th scattering, the $\Lambda_{n}$ is step length, and the $\left|\frac{\mathrm{dE}}{\mathrm{ds}}\right|_{\mathrm{E}_{\mathrm{n}}}$ is the mean energy loss rate which can be obtained from Eq.(6).

Energy deposition distribution (EDD) is an important parameter in consideration of EBL. In order to calculate the energy deposition distribution in resist, we use cylindrical coordination system. We divide the resist layer along z-axis into several thin sub-layers. The EDD was calculated in a radius-depth coordination system, assuming that the scattering to azimuth direction is in symmetry. This means that the resist layer was divided into many concentric rings. The simulation was executed to calculate the total energies $\mathrm{E}(\mathrm{r}, \mathrm{z})$ in every unit ring for EDD function. Using the volume $\Delta \mathrm{V}$ of the sub-layer, the EDD function is given by following equation,

$$
\operatorname{EDD}(r, z)=\frac{E(r, z)}{\left(\Delta V \cdot N_{0}\right)}
$$

where the $\mathrm{N}_{0}$ is total number of incident electron.

\section{Results and Discussion}

\subsection{Description of Electron Scattering Trajectories}

We present Monte Carlo simulation results of energetic electrons impinging in thin film of Calixarene resist based on the model and method as explained above. In the simulation, it was assumed that the electron suffers first scattering after the electron traveling along z-axis for the step-length.

The initial energies of the incident electrons were $30 \mathrm{keV}$ and $10 \mathrm{keV}$. The scattering trajectories of electrons with the incident beam energies in the resist material was used the same as Calixarene resist layer on $\mathrm{Si}$ substrate were shown in Figs.1-2. In the simulation, the thickness of the resist layer of $100 \mathrm{~nm}$, Gaussian beam diameter of $2 \mathrm{~nm}$, and the number of incident electrons of 500 were used. With an incident energy of $30 \mathrm{keV}$, penetration depth was about 4 $\mu \mathrm{m}$ and lateral range was about $1.5 \mu \mathrm{m}$ in Si (Fig.1(a)). In the resist layer, the electron scattering was expanded only to about $20 \mathrm{~nm}$ in radius direction (Fig.1(b)). Although 
using $10 \mathrm{keV}$ incident electrons can diffuse as deep as 0.6 $\mu$ minto the sample, lateral range was expanded to be about $40 \mathrm{~nm}$ in the resist layer which is larger than that of $30 \mathrm{keV}$ (Fig.2(a) and Fig.2(b)). It indicated that as the energy decreases, the electrons scattering range is expanded in the thin resist layer.
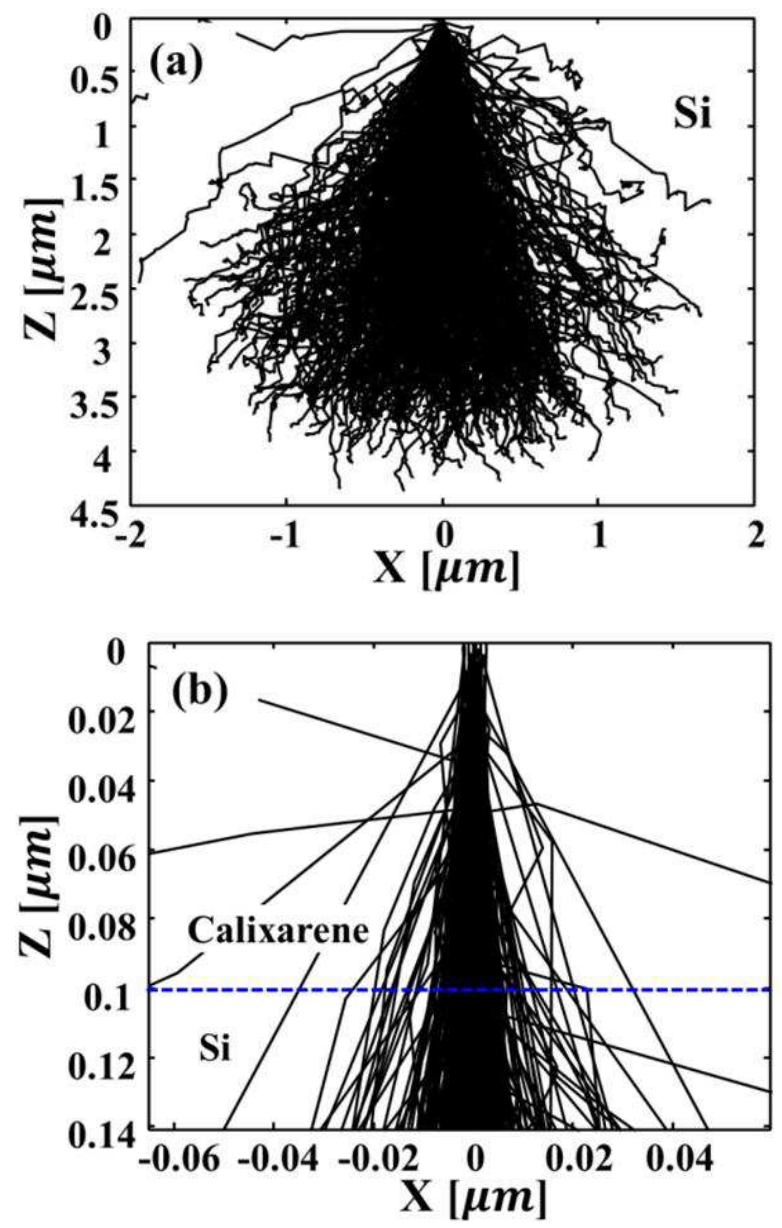

Figure 1.Simulation plot of electron scattering trajectories. (a) Electron scattering trajectories with incident energy $30 \mathrm{keV}$; (b) Trajectories in resist (100nm) with incident energy $30 \mathrm{keV}$.

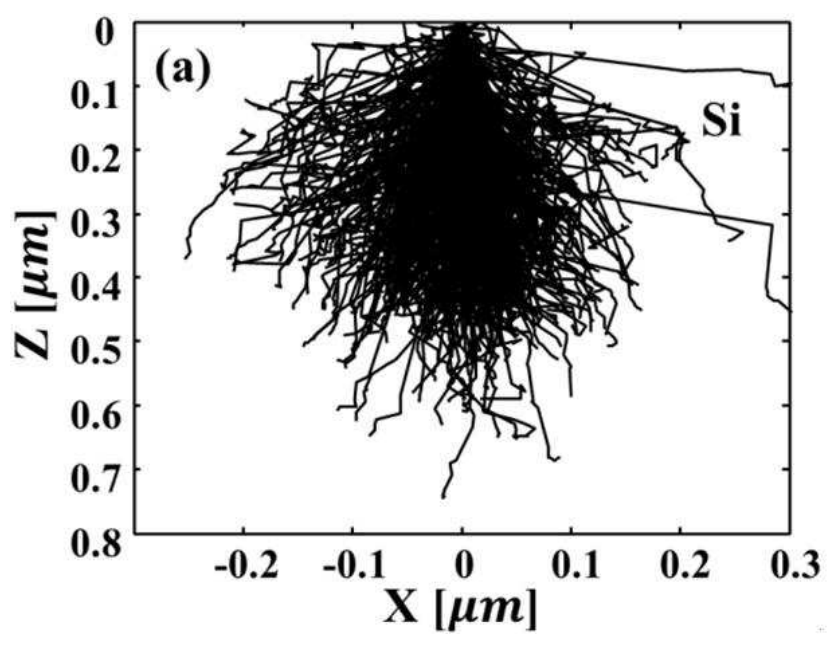

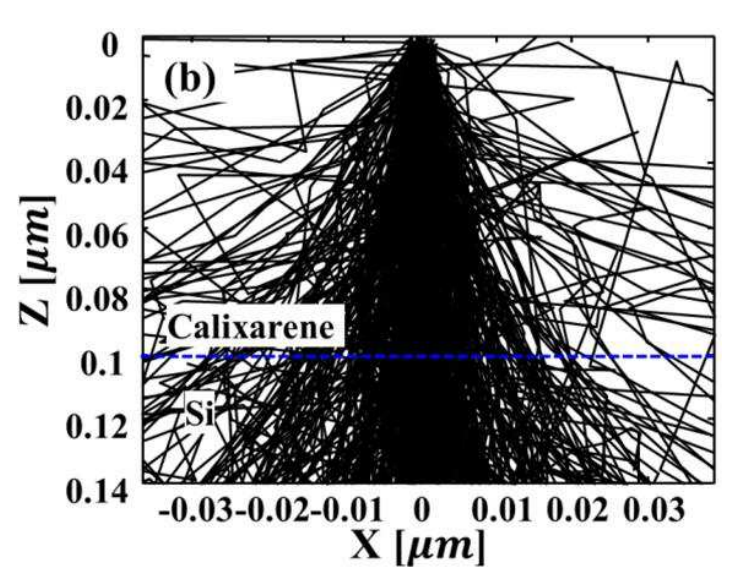

Figure 2.Simulation plot of electron scattering trajectories.(a) Electron scattering trajectories with incident energy 10keV; (b) Trajectories in resist (100nm) with incident energy $10 \mathrm{keV}$.

\subsection{Effect of Beam Diameter on Nano-Sized-Formation}

Beam diameter is one of the major characteristics that affects the accuracy and resolution of EBL systems. Our electron beam writing system can provide us high probe current up to $>2 n A$ at small probe diameter of $<10 \mathrm{~nm}$. In order to study the effect of the Gaussian beam diameter on fine patterning, we have simulated the electron trajectories in resist and EDD distributions when the $\mathrm{s}$ was from $1 \mathrm{~nm}$ to $4 \mathrm{~nm}$, that means the beam diameter was from $2 \mathrm{~nm}$ to $8 \mathrm{~nm}$. In this simulation, the incident electron energy was $30 \mathrm{keV}$, the thickness of resist was $100 \mathrm{~nm}$. Figure 3(a) shows that the scattering area is very narrow that is about $20 \mathrm{~nm}$ in resist using the beam with a very small diameter of about $2 \mathrm{~nm}$. When s increases from $1 \mathrm{~nm}$ to $4 \mathrm{~nm}$, the scattering area becomes large as the beam probe diameter increases (Fig.3.(a)-(d)). Figure 4(a) and Figure 4(b) show the EDD distributions when beam diameters were from $2 \mathrm{~nm}$ to $8 \mathrm{~nm}$ at $10 \mathrm{~nm}$ depth of resist and $30 \mathrm{~nm}$ depth of resist, respectively. They indicated that the smaller the beam diameter, the more centralize the energy deposition distribution. Using high resolution probe beam can effectively to form very fine dot pattern.

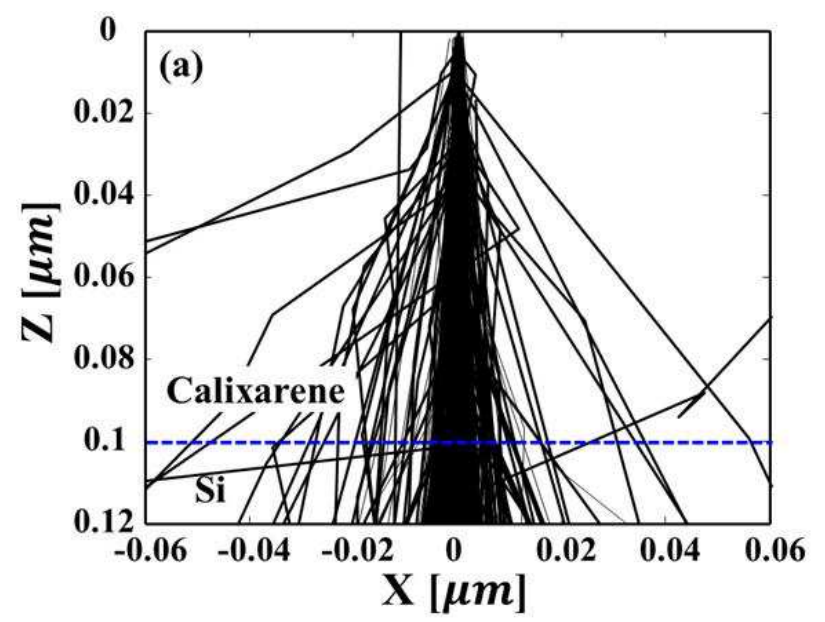



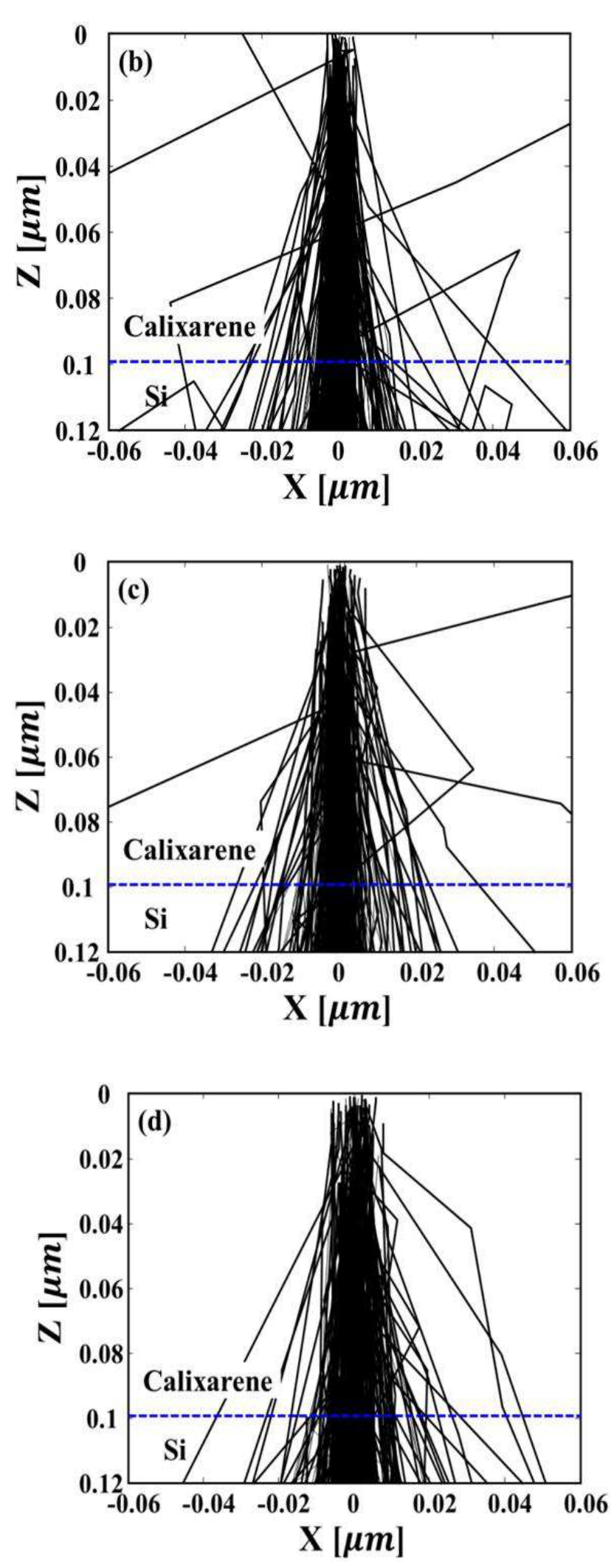

Figure 3.Trajectories in resist(100nm) at various probe diameters. (a) probe diameter $s=1 \mathrm{~nm}$; (b) probe diameter $s=2 \mathrm{~nm}$; (c) probe diameters $=3 \mathrm{~nm}$; (d) probe diameter $\mathrm{s}=4 \mathrm{~nm}$;
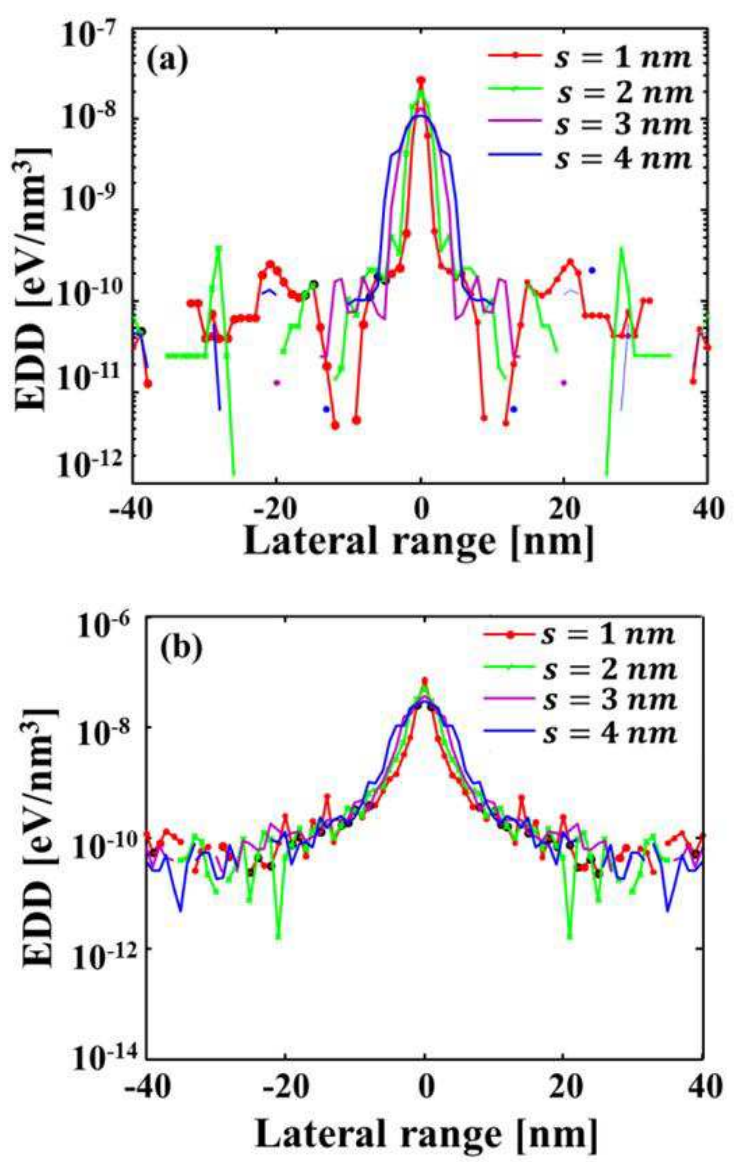

Figure 4.Energy deposition distributions (EDD) at various beam diameters. (a) EDD at various beam diameters in $10 \mathrm{~nm}$ thickness of resist; (b) EDD at various beam diameters in $30 \mathrm{~nm}$ thickness of resist.

\subsection{Energy Deposition Distribution}

The spatial distribution of the deposited energy density in the resist is the most important value that determines the characteristics of the obtained latent image during the electron beam exposure process. ${ }^{21)}$ Here, in order to evaluate the dependence of resist thickness on the formation of very fine dot arrays using e-beam energy of 30 $\mathrm{keV}$, the energy deposition distribution at various depths in thin Calixarene resist was calculated. Figure 5(a) shows the radial distribution of the electron energy deposition in the resist layer of different depths $10 \mathrm{~nm}, 50 \mathrm{~nm}$ and $100 \mathrm{~nm}$ in the case of negative resist Calixarene over a $\mathrm{Si}$ substrate with e-beam energy of $30 \mathrm{keV}$ is obtained following 30000 electrons from a Gaussian beam with a probe diameter of 2 $\mathrm{nm}$. It can be clearly seen that the shallower the depth from the surface of the resist, the narrower and the sharper the distribution. From the energy deposition distribution, we can obtain the relationship between thickness of resist and the dot diameter in various critical energies, as shown in Fig.5(b). The figure shows that thickness of resist less than $30 \mathrm{~nm}$ can form the sub-10nm dot pattern while the thickness of resist of less than $20 \mathrm{~nm}$ can obtain very fine dot with a size of $5 \mathrm{~nm}$. Therefore, the resist film is used must be as thin as possible for very fine dot array formation. 

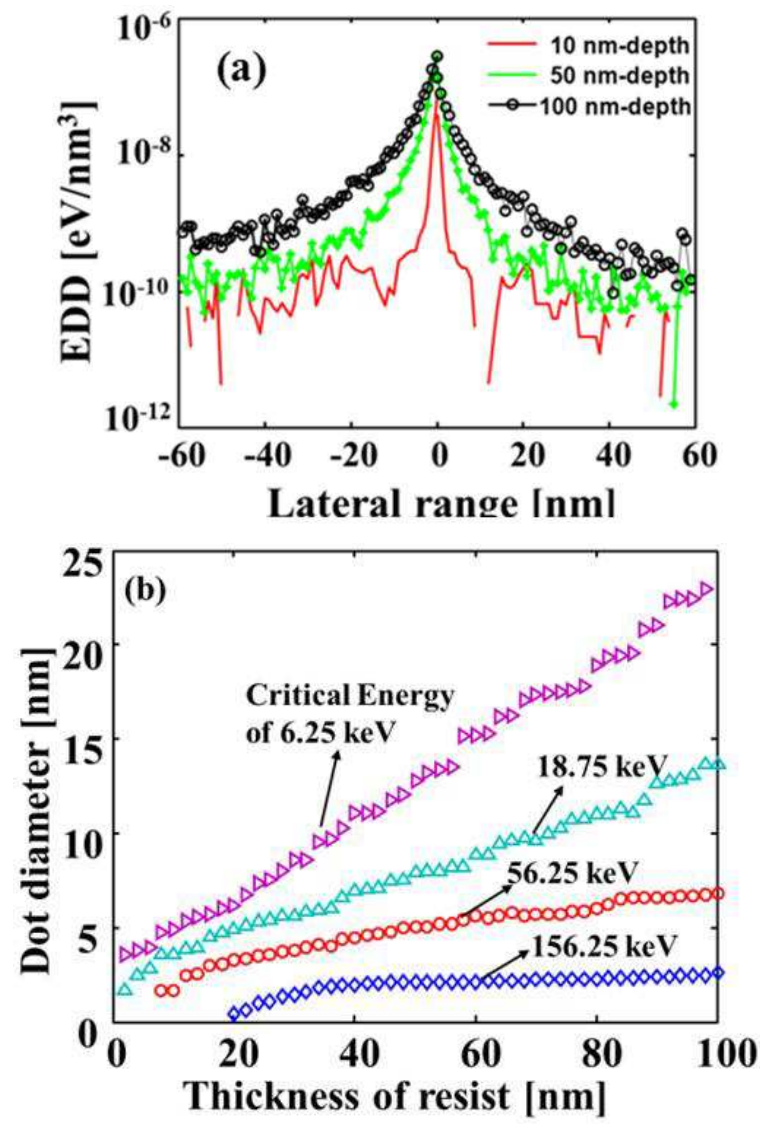

Figure 5. (a) Energy deposition distribution of different depths of resist; (b) the relationship between the thickness ofresist and dot diameter at different critical energy.

\subsection{Consideration for Resist Development Based on the $E D D$}

The goal of the simulation of the processes in EBL is prediction of the resist profile of the developed exposed microstructure. Resist development is defined as the resist molecule is solved and linked at critical energy density in positive and negative resists, respectively. For the evaluation of the dot size in resist profile, calculations in a wide range of critical energy densities were executed. Figures 6.(a)-(d) show the developed resist profiles at various critical energy densities of $156.25 \mathrm{keV} / \mathrm{cm}^{3}-$ $6.25 \mathrm{keV} / \mathrm{cm}^{3}$ in Calixarene resist. It is clear that the dot diameter decreases with not only thickness but also critical energy. Small pattern formation is possible by selecting the higher critical energy density, which corresponds to exposure dosage in experiment.

According to the negative resist development mechanism, the exposed resist portions are remained on the substrate. Although the height of the resist pattern is not complete and short, when the critical energy density of $156.25 \mathrm{keV} / \mathrm{cm}^{3}$, the pattern of $3 \mathrm{~nm}$ can be formed as shown in Fig.6(a). It is clear that the smaller pattern size is obtained by selecting the higher critical energy density, but the height of the resist pattern decreases as the critical energy density increases.

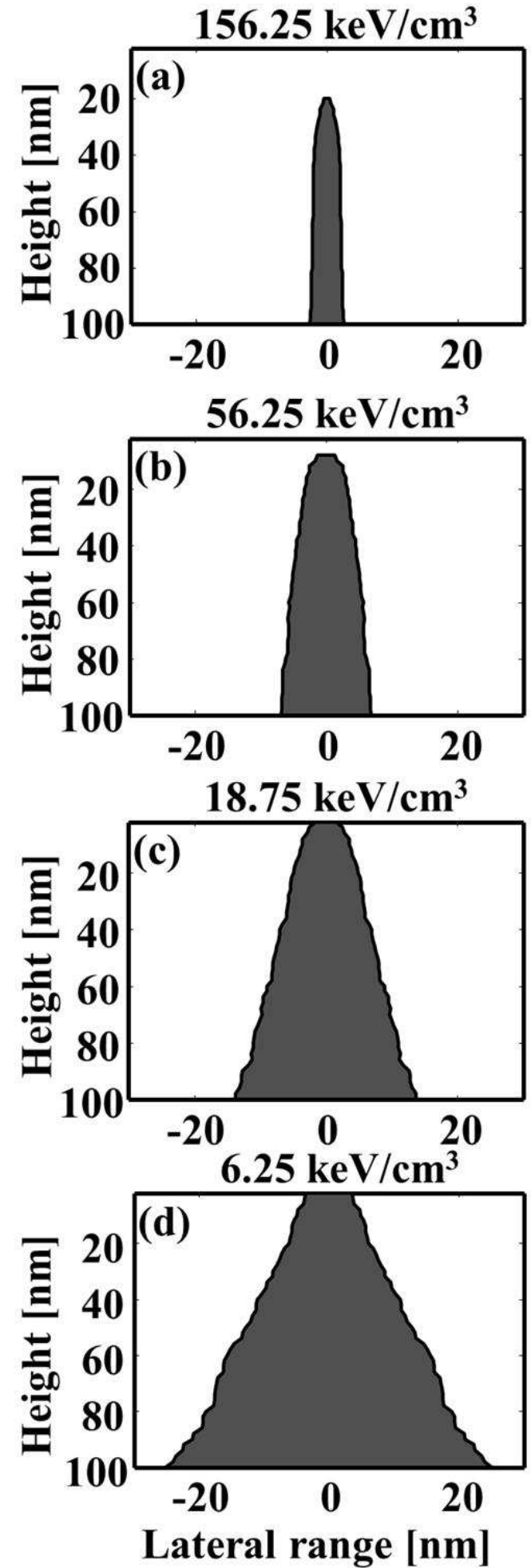

Figure 6. Simulated resist profiles at various critical energies.(a) Critical energy at $156.25 \mathrm{keV} / \mathrm{cm}^{3}$; (b) Critical energy at $56.25 \mathrm{keV} / \mathrm{cm}^{3}$; (c)Critical energy at $18.75 \mathrm{keV} / \mathrm{cm}^{3}$; (d) Critical energy at $6.25 \mathrm{keV/}$ $\mathrm{cm}^{3}$.

\subsection{Comparision of Calixarene Resist with PMMA Resist}

PMMA $\left(\left(\mathrm{C}_{5} \mathrm{H}_{8} \mathrm{O}_{2}\right)_{n}\right)^{22)}$ as a positive resist is a compound of carbon $(\mathrm{C})$, hydrogen $(\mathrm{H})$, and oxygen $(\mathrm{O})$ with a molecular weight of 100.067 having average density 
$1.19 \mathrm{~g} / \mathrm{cm}^{3}$, however, Calixarene $\left(\mathrm{C}_{36} \mathrm{H}_{36} \mathrm{O}_{4} \mathrm{Cl}_{4}\right)$ is roughly a ring-shaped molecule with a diameter of less than $1 \mathrm{~nm}$ and thus is mono-dispersed with a molecular weight of 674 and having density $1.09 \mathrm{~g} / \mathrm{cm}^{3} .^{23-24)}$ We use the random number method to select the scattering atom, and we can get that the total cross section in PMMA is larger than that inCalixarene. Consequently, the scattering lateral range of about $40 \mathrm{~nm}$ in PMMA resist is larger than that about 20 $\mathrm{nm}$ in Calixarene resist by using $30 \mathrm{keV}$ incident energy. Furthermore, because the basic component of Calixarene is a phenol derivative from the strong chemical coupling of the benzene ring, which seems Calixarene has high durability and stability. Therefore, Calixarene negative resist is very suitable to form nanometer-sized dot.

\section{Conclusions}

Using home-made Monte Carlo simulation program with Gaussian electron beam and Calixarene negative resist, we calculated electron interactions in sample at various conditions. In the simulation, we calculated energy deposition distributions in the sample of 100-nm-thick Calixarene resist layer on silicon substrate when $30 \mathrm{keV}$ electrons impinge into the resist layer on $\mathrm{Si}$ substrate. The evaluation of dot size of resist profile was studied as a function of incident energy, Gaussian beam diameter and resist thickness, theoretically. As a result, the followings conclusions can be drawn:

(1) It is necessary to use the thinner resist film for the formation of very fine dots using Gaussian beam with fine probe diameter of $2 \mathrm{~nm}$.

(2) It is potential to form $3 \mathrm{~nm}$-diameter dotwhen the resist thickness is less than $20 \mathrm{~nm}$ using negative resist Calixarene by the simulation.

(3) High critical energy density is suitable to form very fine dot pattern.

(4) Increasing of incident electron energy can also make it easy to form smaller pattern.

(5) Calixarene negative resist has an ability to make smaller pattern comparing with PMMA positive resist.

\section{References}

[1] Y.Nakamura,T.Sakamoto, and J.S.Tsui: Jpn. J. Appl. Phys., Vol. 34 (1995) 4562

[2] C.Joachim, J.K.Gimzewski, and A.Aviram: Nature Vol. 408 (2000) $541-548$
[3] S.Hosaka, T.Akahane, M.Huda, T.Tamura, Y. Yin, N. Kihara, Y. Kamata and A. Kitsutsu:Microelectron. Eng., Vol. 88 (2011) 2571-2575

[4] S.Hosaka, H.Sano, K.Itoh and H. Sone: Microelectron.Eng. , Vol. 83 (2006) 792-795

[5] Z.Mohamad, M.Shirai, H.Sone, S.Hosaka and M.Kotera, Nanotechnology,Vol. 19 (2008)025301 1-4

[6] B.D.Terris,T.Thomson, and J.Phy.D: Appl. Phys., Vol. 38 (2005) R199-R222

[7] I.Bita, J.K.W.Yang, Y.S.Jung, C.A.Ross, E.L.Thomas and K. K. Berggren, Science Vol. 321 (2008) 939-943

[8] Z.Chen, Y.-M.Lin, M.J.Rooks, and P.Avouris: Physica E (Amsterdam), Vol.40 (2007) 228-232

[9] A.A.Tseng, K.Chen, C.D.Chen, and K.J.Ma: IEEETrans. Electron. Packag. Manuf. Vol. 26 (2003) 141-149

[10] M. Kotera, and T. Maekawa: Jpn. J. Appl. Phys.,Vol. 48 (2009) 06FB05-1 - 06FB05-4.

[11] J.Fujita, Y.Ohnishi, Y.Ochiai, and S.Matsui: Appl. Phys. Lett., Vol. 68 (1996) 1297-1299

[12] S.Hosaka, Y.Tanaka,M.Shirai, Z. Mohamad and Y. Yin: Jpn.J.Appl.Phys., Vol. 49, (2010)046503 1-3

[13] M.Kotera, S. Yamaguchi, S. Umegaki, and H. Suga:Jpn. J. Appl. Phys., Vol. 33(1994) 7144-7147

[14] H.G.Duan, D.Winston, J.K.W.Yang, B.M.Cord, V. R. Manfrinato and K. K. Berggren: J. Vac. Sci. Technol., Vol.28 (2010) C6C58

[15] T.H.P.Chang: J. Vac. Sci. Technol., Vol. 12 (1975) 1271-1275

[16] K. Murata, and T.Matsukawa: Jpn.J.Appl.Phys., Vol. 10, 678-685 (1971)

[17] N.Aizaki: Jpn.J.Appl.Phys., Vol. 18 (1979) 319-325

[18] M. Kotera, K. Murata, and K. Nagami:J. Appl. Phys., Vol. 52, (1981)7403

[19] M. Kotera: J. Appl. Phys., Vol. 65, (1989)3991

[20] D.C.Joy and S.Luo: Scanning, Vol. 11 (1989) 176

[21] K.Vutova, G.Mladenov,I.Raptis, A. Olziersky: J.Mater.Process.Technol.,Vol. 184, 305-311 (2007)

[22] M.S.Singh, R.K.B.Singh, R.Khatri., and B.I.Sharma: Adv. Sci. Lett., Vol. 3 (2010) 57-61

[23] M.Ishida, J.I.Fujita, T.Ogura, Y. Ochiai, E. Ohshima: Jpn. J. Appl. Phys., Vol. 42, 3913-3916 (2003)

[24] P.Jedrasik, M.Hanson, and B.Nilsson: Microelectron.Eng., Vol. 53 (2000) 497-500 